\title{
DEVENIR ET AVENIR \\ DE LA BILHARZIOSE EN TUNISIE
}

\author{
Par Alexis COUMBARAS
}

L'étude détaillée, région par région, de la bilharziose vésicale en Tunisie, a été faite antérieurement (1). Il s'agit d'une endémie en foyers disséminés atteignant 50 pour cent de la population de ces foyers.

Nous voudrions ici faire la synthèse de nos observations sur l'écologie de la faune malacologique, une étude comparative des différents types d'habitat des mollusques vecteurs, de la transformation de ces gîtes, des incidences qui en dérivent sur l'évolution de l'endémie bilharzienne, et sur les moyens de lutte qu'il y aurait lieu de mettre en œuvre.

Il convient de souligner, dès le début, le caractère limité de l'endémie bilharzienne en Tunisie (voir carte) : la maladie n'affecte que la zone subsaharienne du pays. Par ailleurs, en raison même du caractère pré-désertique de la région, la bilharziose ne sévit que dans des foyers bien délimités de cette région, séparés par des étendues désertiques non habitées de manière permanente et dépourvues d'eau. Cela ne signifie évidemment pas, qu'au sein de la zone intéressée, toute collection d'eau recèle nécessairement des bulins. Il existe des sources d'eau hypersalée, sulfureuse ou thermale, où $B$. truncatus, seul vecteur de bilharziose en Tunisie, ne saurait proliférer. Par ailleurs, en raison de la forte salinité du terrain dans ces régions, toute eau stagnante se charge très rapidement en sel et devient impropre à abriter des bulins. C'est le cas des eaux du Chott el Djerid, du Chott el Fedjedj, d'autres chotts moins importants et de leurs innombrables prolongements qui servent de déversoirs aux oueds qui en sont tributaires lors des périodes de crues. La salinité de ces eaux, qui s'étendent en vastes nappes boueuses, est de l'ordre de 15 g./1. de CINa. Il en est de même des eaux de drainage constituées par des eaux résiduelles provenant d'un réseau d'irrigation, eaux qui n'ont pas été absorbées lors du parcours du périmètre irrigué, qui se sont chargées en éléments minéraux et qui, à l'issue de ce périmètre, présentent une salinité de l'ordre de $7 \mathrm{~g} . / 1$.

Or, une salinité de $4 \mathrm{~g}$./1. est mortelle pour les bulins à cent pour cent et en 24 heures. Il est évident que de telles collections d'eau ne sauraient jamais présenter un danger bilharzien. C'est là une différence importante d'avec ce 
qui se passe dans d'autres pays, où les eaux de drainage constituent des gîtes de première importance, et où les eaux stagnantes ou peu courantes sont également infestées de mollusques. En Tunisie, on ne trouve jamais de bulins dans des eaux stagnantes. Par ailleurs, en règle générale, un point d'un cours d'eau donné est d'autant moins susceptible d'abriter des bulins, qu'il est situé à une grande distance en aval de la source, et ce, en raison de la charge en sel de l'eau qui augmente avec la distance parcourue en terrain salé.

Enfin, dans de très nombreux cas, il semble que toutes les conditions favorables à l'établissement d'une colonie de bulins soient réunies pour un point d'eau donné, ou que tout au moins on ne puisse déceler un facteur interdisant leur prolifération. Et pourtant, ce point d'eau reste indemne de bulins. De nombreux auteurs, dans de nombreux pays, ont cherché les facteurs déterminants de la prolifération des mollusques sans arriver à des résultats probants. On a évoqué le rôle de facteurs physiques: températures de l'eau, ensoleillement, humidité, rapidité du courant ; de facteurs chimiques : teneur en différents ions et en matières organiques; de facteurs biologiques enfin : abondance et qualité de la végétation aquatique, présence ou absence d'animaux prédateurs, fréquentation importante ou non du point d'eau par des usagers humains ou animaux.

Il nous semble que, si chacun de ces facteurs peut être inhibiteur par excès ou par défaut, aucun de ces facteurs pris séparément, ni même la réunion de tous ces facteurs dans leur expression optima ne suffisent à expliquer la présence de bulins dans un point d'eau, puisqu'on trouve beaucoup de points d'eau donnant satisfaction à toutes ces conditions, et où les bulins refusent de proliférer. Nous venons de voir les aspects limitatifs de la salinité d'une eau quant à sa capacité d'héberger des bulins. On conçoit également que ces derniers ne puissent guère proliférer dans une eau thermale à $45^{\circ}$, comme il y en a dans le Sud tunisien, ou dans une conduite d'eau entièrement recouverte, mais là encore température et obscurité interviennent de manière inhibitrice et il en est de même de tous les autres facteurs \& déterminants » et * favorisants » décrits à ce jour, qui ne sont en fait que des facteurs inhibiteurs au-delà de certains seuils d'inhibition. La connaissance de ces facteurs est évidemment utile, mais ne saurait expliquer dans tous les cas la présence ou l'absence de bulins dans un point d'eau donné, puisque, dans un point d'eau, satisfaisant à ces différents facteurs, il peut $\mathrm{y}$ avoir ou ne pas $\mathrm{y}$ avoir de bulins, et ce, pour des motifs qui demeurent jusqu'à ce jour totalement inconnus.

$\mathrm{Si}$ les raisons premières qui régissent l'existence d'un peuplement malacologique nous échappent, on peut cependant décrire et classer les points d'eau susceptibles d'héberger des bulins. On peut suivre les modifications de la faune malacologique en fonction des modifications hydrauliques des gîtes, et en tirer parti pour la lutte contre les mollusques.

On peut distinguer, sous l'angle de leur rôle en tant que foyers infestants, les gîtes ancestraux, anciens et récents. Nous n'ignorons pas ce qu'une telle 
classification comporte d'arbitraire ; cependant, elle a l'avantage de faire ressortir nettement le caractère évolutif des conditions épidémiologiques de la bilharziose en Tunisie, de faire ressortir également le rôle dominant des modifications apportées par l'homme à l'hydraulique naturelle. Enfin, il est à noter que si ces trois types de gîtes portent des noms qui les situent dans le temps, les uns par rapport aux autres, il s'agit cependant d'entités bien individualisées, sans formes intermédiaires de passage de l'une à l'autre, sans aucune solution de continuité entre elles.

Les gîtes ancestraux sont les seuls qui ne doivent rien à la main humaine, étant constitués par des points d'eau naturels, et qui demeurent inchangés à nos jours. Il est tout naturel d'admettre que la propagation des bulins aux deux autres types de gîtes s'est faite à partir de ces réservoirs primaires de l'espèce, à moins que l'on n'admette que les bulins n'ont fait leur apparition en Tunisie qu'après que l'homme ait commencé à pratiquer la culture irriguée, ce qui, a priori, paraît peu probable.

Les gîtes anciens sont ceux artificiellement créés par l'homme à la faveur de pratiques agricoles d'irrigation, telles qu'elles sont pratiquées depuis certainement fort longtemps.

Les gîtes récents sont également de création humaine, mais ne remontent qu'à quelques années. Par leur nature très différente des précédents et les problèmes nouveaux qu'ils posent, ils méritent d'être réunis dans un troisième groupe distinct et d'être étudiés séparément.

\section{I. - Gîtes ancestraux}

Ces gîtes sont constitués par deux types de collections d'eau : les oueds et les sources oasiennes.

$1^{\circ}$ Les oueds (photo 1) sont des cours d'eau à débit extrêmement irrégulier. La plupart sont complètement à sec pendant toute l'année, hormis en période de pluie au printemps et surtout en automne, où on assiste à des crues brutales et fugaces. Il est évident que de tels oueds, ne contenant de l'eau que quelques jours, au maximum quelques semaines par an, ne sauraient héberger des mollusques. Il existe cependant quelques oueds à débit également extrêmement irrégulier, puisqu'ils subissent le même régime pluviométrique, mais qui ne se dessèchent jamais complètement en raison de l'existence de sources artésiennes qui s'ouvrent au fond de leur lit. Ces sources ont un débit très faible en règle générale, insuffisant, par suite de la réinfiltration et de l'évaporation, pour entretenir un ruissellement continu au fond du lit de l'oued. Mais elles suffisent pour entretenir de place en place, dans les parties déclives, des collections d'eau de quelques mètres de diamètre et de moins d'un mètre de profondeur, lentement mais constamment renouvelée. De telles collections d'eau constituent parfois des gîtes à bulins et des sources d'infestation bilharzienne à la suite de baignades (enfants surtout) et d'usages domestiques (femmes surtout) de 
ces eaux. Ces eaux ne sont pas utilisées à des fins agricoles, leur point d'émergence étant situé plus bas que les terrains à irriguer.

D'une manière générale, ce type de foyer infestant est rare dans le Sud tunisien. Il est cependant responsable des foyers de bilharziose d'El-Hamma, Matmata et Zarat (voir carte), où se maintient un taux d'infestation relativement faible, respectivement $29,8,5$ et 11 pour 100 dans une population de 100 enfants d'âge scolaire prise au hasard, et où l'atteinte des adultes est beaucoup plus faible, sans doute à la suite de la non-utilisation à des fins agricoles de ces eaux.

$2^{\circ}$ Les sources oasiennes: Il s'agit d'un type de gîte beaucoup plus répandu, responsable d'endémies massives, avec taux d'infestation de l'ordre de 60 pour 100 dans les villages de la région du Nefzaoua où ce type de gîte prédomine.

Ces sources, disséminées dans une région particulièrement aride, sont constituées par un jaillissement artésien d'un débit de l'ordre de quelques litres par seconde, dépassant rarement $10 \mathrm{l} . / \mathrm{sec}$.

Elles se présentent comme des collections d'eau de quelques mètres de diamètre, de quelques centimètres à 2 mètres de profondeur. Au fond d'une dépression en vasque creusée dans le sable, on remarque un bouillonnement à l'endroit où jaillit l'eau (photo 2).

Tout autour de la source, profitant de l'apport d'eau, poussent des palmiers, réalisant ainsi un aspect d'oasis typique d'une grande beauté. L'ensemble se présente ainsi comme une cuvette creusée dans le sable, dont le fond contient de l'eau et les bords portent une couronne de palmiers.

Chaque source ne garde pas indéfiniment un statut immuable quant à son débit et son aspect extérieur. Indépendamment de toute intervention de l'homme, ces sources sont l'objet d'une lente évolution naturelle.

Les études hydrogéologiques entreprises dans la région ont démontré l'abaissement du niveau statique de la nappe d'eau souterraine. Certains auteurs expliquent par cet abaissement le fait que la contrée autrefois fertile est devenue semi-désertique. Plus près de nous, les Romains ont réalisé des aménagements et constructions hydrauliques qui n'auraient guère pu fonctionner avec le niveau statique actuel de la nappe.

Cette évolution à long terme joue évidemment dans le sens d'une diminution du débit, d'un asséchement graduel des sources oasiennes, mais il existe également un autre mécanisme agissant dans le même sens et de manière beaucoup plus rapide : c'est le processus de l'ensablement continuel des sources. Ensablement d'origine éolienne tout d'abord. Il vente beaucoup et souvent dans les régions pré-sahariennes, et les grains de sable transportés par le vent ne sont que partiellement et momentanément arrêtés par les palmiers qui poussent autour de la source. Ensablement par le fond aussi : le jaillissement artésien entraîne avec lui, depuis les profondeurs, des grains de sable qui sont ensuite abandonnés au fond de la cuvette. 
L'évolution naturelle des sources oasiennes se fait donc vers l'ensablement et le tarissement. Aussi, rencontre-t-on dans la région tous les stades de cette évolution : sources à débit relativement important, pouvant suffire à l'alimentation en eau de quelques palmiers poussant dans la proximité immédiate, sources plus ou moins transformées en marécages; enfin, une cuvette creusée dans le sable, avec un fond desséché et une maigre couronne de palmiers en train de dépérir, marque parfois l'emplacement passé d'une source. Le processus est évidemment assez lent, mais peut s'accomplir parfois au cours d'une ou de deux générations de cultivateurs, qui parlent de « naissance ", « vieillesse » et « mort » d'une source pour désigner les différentes phases de l'évolution que nous venons de décrire et qu'ils arrivent à observer au cours de quelques décades.

$\mathrm{La}$ faune malacologique ne manque pas d'être influencée par cette évolution de son biotope. Bien avant l'asséchement total d'une source, les bulins deviennent de moins en moins nombreux et finissent par disparaître complètement. Il faut être très prudent avant d'affirmer qu'un gîte connu pour avoir abrité des bulins a définitivement été abandonné par les mollusques. Il ne faut évidemment pas prendre pour une désertion définitive d'un gîte la disparition saisonnière des bulins de novembre à avril. C'est là un phénomène constant en Afrique du Nord (2). Pas plus qu'ailleurs, le sort des mollusques pendant leur hibernation n'a pu être déterminé avec précision. Hibernation à l'état d'œufs, sans doute, à en juger d'après le grand nombre de petits mollusques fraîchement éclos au printemps; mais nous avons pu voir également, rarement il est vrai, de très gros spécimens $(11 \mathrm{~mm}$.) accrochés à des plantes aquatiques en plein mois de janvier.

Il faut se souvenir également qu'un curage détruit une proportion importante de mollusques, au point de faire croire à un abandon définitif du gite par les bulins. On trouvera cependant dans les boues de fond, abandonnées sur les berges lors du curage, un grand nombre de tests vides, qui nous prouvera que le dépeuplement du gîte n'est que momentané.

Par contre, il nous a été possible d'affirmer à la suite de comparaisons avec des observations antérieures $(3,4$ et 5$)$, portant sur des gîtes bien identifiés de la région de Gafsa, l'extinction progressive de ces foyers. Il s'agit de sources oasiennes qui ont été suivies pendant 40 ans par différents auteurs qui les ont décrites avec suffisamment de précision pour qu'on puisse les identifier exactement sur le terrain, et comparer leurs données hydrographiques et leurs peuplements malacologiques passés et actuels. Il ressort de cette étude que :

1) le débit de ces sources a progressivement diminué suivant les mécanismes décrits plus haut ;

2) le peuplement malacologique a progressivement diminué jusqu'à la disparition totale des bulins à l'heure actuelle dans certains de ces gites;

3) sans que l'étude des caractéristiques physiques et chimiques de l'eau puisse expliquer cette disparition. 
En creusant dans les versants des points d'eau, signalés autrefois comme gîtes à bulins importants, et actuellement abandonnés par les mollusques, nous avons toujours trouvé de nombreux tests de bulins. Ces tests étaient intimement incorporés à la roche marneuse ambiante, et non à de la boue de curage. Ils doivent être regardés comme des fossiles, témoins d'un peuplement ayant été important jadis et qui s'est éteint depuis. Il n'a pas été possible de définir la cause de ce dépeuplement. Le débit des sources avait certes diminué, ainsi que l'espace vital mis à la disposition des bulins, mais les données physiques, chimiques, et la végétation aquatique paraissaient être parfaitement compatibles avec la vie des mollusques, et tout à fait semblables à celles de gites voisins, continuant à abriter des bulins. Cette observation ne fait que confirmer ce que nous avons dit plus haut: les facteurs déterminants de la prolifération des bulins dans la nature restent encore à trouver.

Les sources qui viennent d'être décrites constituent de très importants foyers d'infestations. Pendant les fortes chaleurs de l'été, ces sources servent de baignades à la population adulte autant qu'à celle des enfants.

L'eau infestée de cercaires est utilisée à des fins agricoles, d'où contamination professionnelle des cultivateurs. C'est toujours la même eau qui est utilisée à des fins ménagères, notamment pour le lavage du linge, manière classique de contamination pour les femmes. Enfin, c'est toujours la même eau qui sert de boisson, ce qui constitue un mode de plus de s'infester pour tout le monde.

\section{II. - Gîtes anciens}

Dans un pays où la pluviométrie est faible (de l'ordre de $70 \mathrm{~mm}$. par an) et irrégulière, l'irrigation est la seule méthode garantissant un apport d'eau suffisant et régulier. Aussi, les sources oasiennes sont-elles largement exploitées à des fins agricoles. L'utilisation de leur eau est opérée selon des procédés qui n'ont guère varié depuis des siècles, qui ont donné lieu à la création par l'homme de gîtes à bulins tout aussi anciens que les sources elles-mêmes, et qui suivent ces sources dans leur évolution.

L'irrigation à partir des sources oasiennes se fait d'une manière intermittente. On laisse s'accumuler l'eau pendant un ou plusieurs jours dans la cuvette. Une fois qu'il y en a suffisamment, une brèche est ouverte dans l'un de ses versants, et l'eau s'écoule rapidement dans une rigole (séguia) qui lui fait suite, puis dans les canaux secondaires qui en dérivent et leur ramification, atteignant ainsi les secteurs les plus éloignés du périmètre irrigué. Une fois toute l'eau écoulée, on colmate la brèche et on attend que la cuvette se remplisse à nouveau pour recommencer l'opération. L'irrigation intermittente permet ainsi un apport d'eau important, quoique périodique, aux différents secteurs du périmètre irrigué. Avec des débits aussi faibles que ceux dont nous parlons, l'irriga- 
tion continue serait impraticable, car, au fur et à mesure de son lent écoulement, l'eau serait entièrement réabsorbée par le sol et évaporée dans l'atmosphère, bien avant d'atteindre les secteurs quelque peu éloignés de la plantation (photo 3).

Les séguias et leur ramification ne contiennent de l'eau que de manière périodique et pendant un laps de temps assez court. Il n'empêche qu'elles constituent souvent des gîtes à bulins de premier ordre et des foyers d'infestation importants. En effet, les bulins s'accommodent très bien de l'humidité résiduelle du fond des séguias pendant les périodes de vacuité.

Toutes les séguias ne contiennent pas des mollusques, et il est fréquent de ne pas en trouver dans une séguia, alors que la source qui en est tributaire constitue un gîte important de bulins. D'une manière générale, on trouve des bulins plutôt dans les grosses séguias primaires et leurs premières ramifications. Les chances de trouver des bulins diminuent au fur et à mesure qu'on s'éloigne de la source. Ce phénomène s'explique aisément dans les terrains salés : l'eau se charge en sel au fur et à mesure qu'elle parcourt son trajet, et devient de moins en moins apte à servir d'habitat aux mollusques. Cependant, le fait est observé également dans les terrains peu salés. Là aussi, on rencontre d'abondantes colonies de bulins dans les grosses séguias primaires, à débit relativement important, et on n'en trouve pas dans les petits canaux terminaux. La notion d'abondance de l'eau en tant que facteur favorable à la prolifération des bulins se retrouve d'une manière constante dans le Sud tunisien, sans que nous puissions nous en donner une explication rationnelle par l'analyse des données physiques, chimiques, biologiques concernant ces gîtes qui ne diffèrent souvent pas de celles qui caractérisent des collections d'eau plus réduites.

La contamination à partir de ces gîtes est évidemment surtout liée aux travaux agricoles. Les ablutions rituelles des pieds chez les musulmans, ainsi que quelquefois la consommation de ces eaux, jouent également un rôle.

Ce qui a été dit pour les sources oasiennes, en ce qui concerne leur pérennité relative en tant que gîtes à bulins, est également valable pour ces gîtes artificiels, de création ancienne.

Il est vraisemblable que, depuis que les pratiques agricoles qui viennent d'être décrites existent - et elles existent certainement depuis fort longtemps - les séguias constituent des foyers d'infestation bilharzienne. Dans les séguias dont le débit d'eau a baissé par suite d'une baisse du débit de la source tributaire, nous avons constaté, comme pour les sources, la disparition des bulins dont la présence avait été notée par des observateurs qui nous avaient précédé sur les lieux il y a quelques décades (3, 4 et 5). Là encore, nous avons trouvé, en creusant dans les versants de ces séguias, de nombreux bulins fossiles témoignant d'un peuplement passé de ces gîtes, actuellement abandonnés. Là encore, nous n'avons pu déterminer les raisons intimes de la disparition des mollusques, tout en notant qu'elle accompagne là aussi une diminution du débit. 


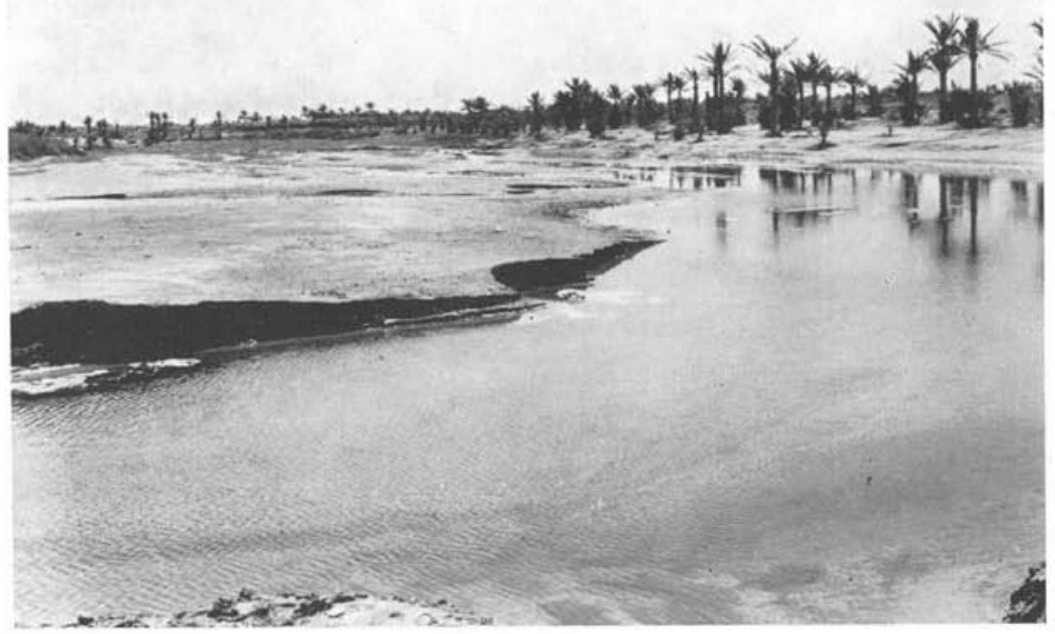

Рното 1. - Un oued en période de crue. En période normale, seules subsistent quelques collections d'eau dans les parties les plus déclives du lit, alimentées par des petites sources s'ouvrant dans le lit même de l'oued

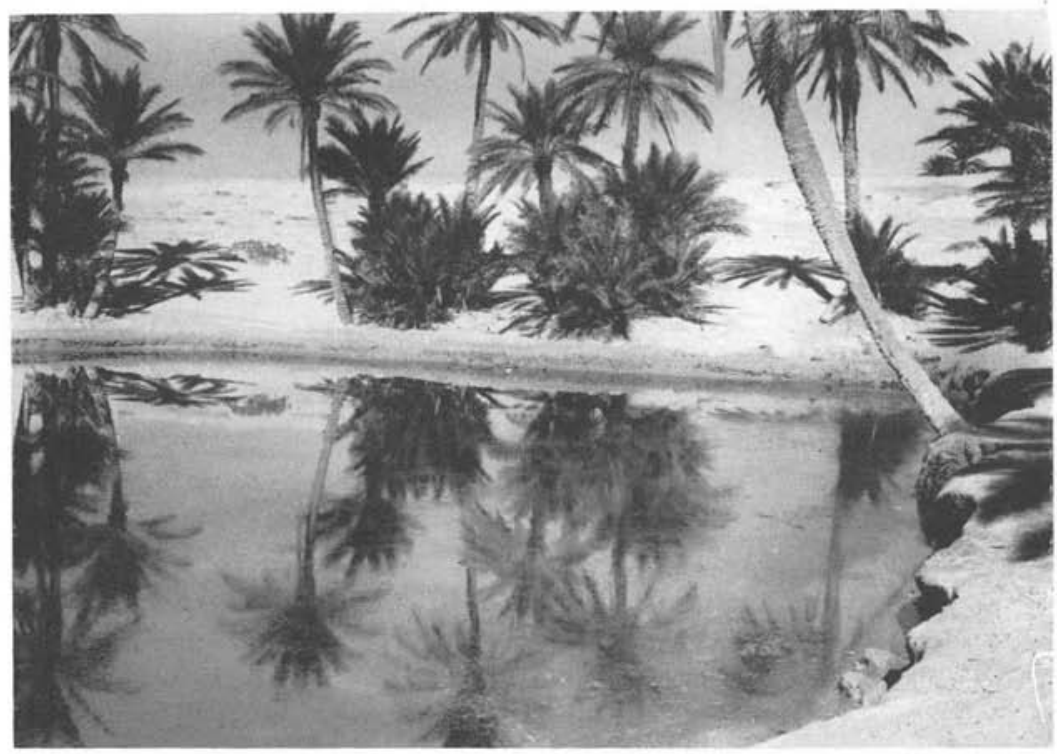

Рното 2. - Une source d'oasis typique 


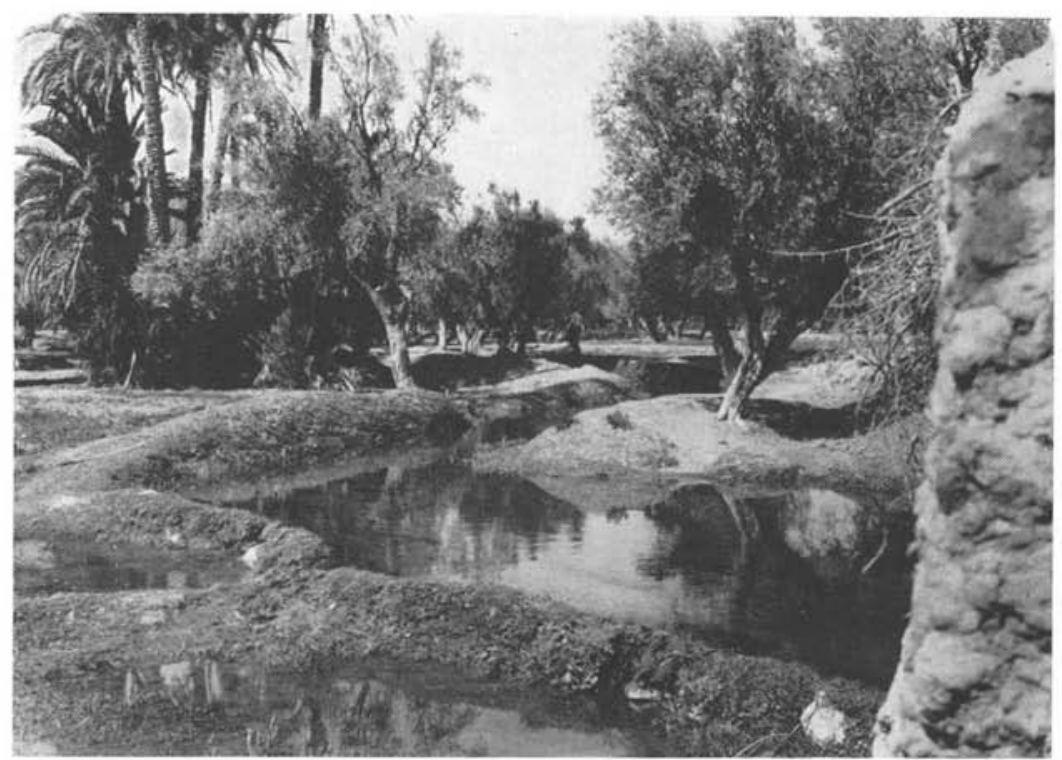

Рното 3. - Séguia principale de grande dimension irriguant palmiers et oliviers

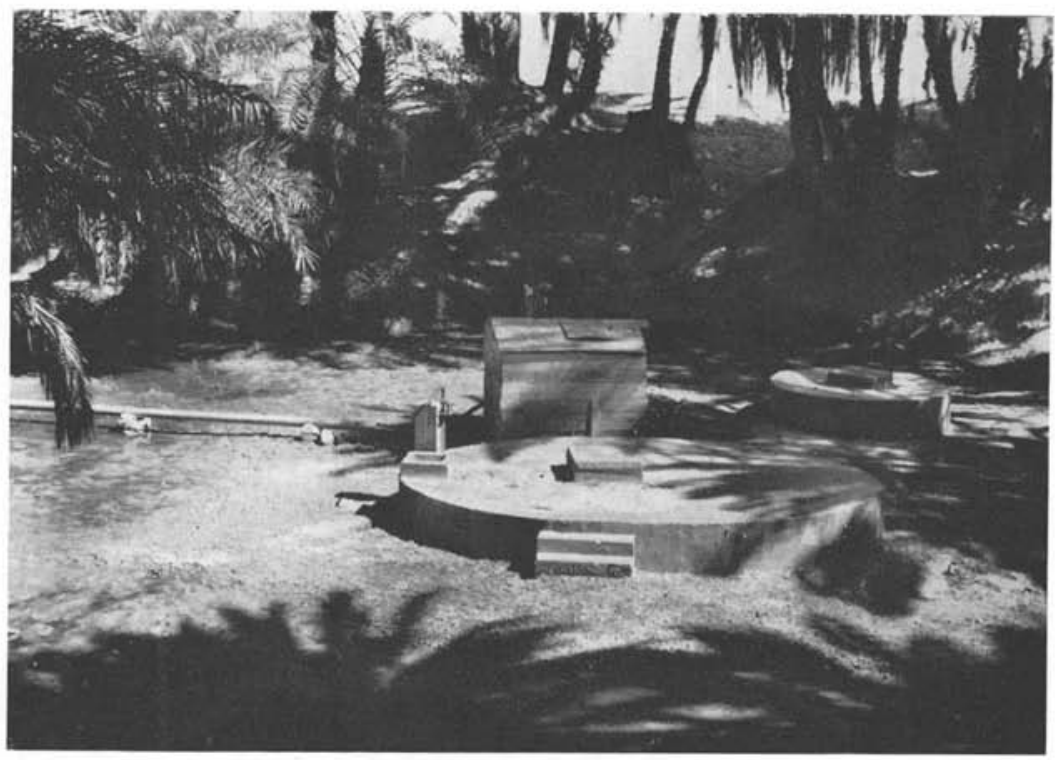

Рното 4. - Le captage d'une source. On reconnaît l'aspect familier de la dépression en cuvette d'une source oasienne, avec sa couronne de dattiers qui poussent sur les bords, telle qu'elle se présente sur la photo 2 . Les griffons de la source sont emprisonnés sous deux buses en béton, dont on voit le plafond circulaire, et l'eau amenée aux terrains à irriguer par une conduite aérienne fermée (à gauche). Le fond de la source est à sec; un important gîte à bulins a été définitivement éliminé 


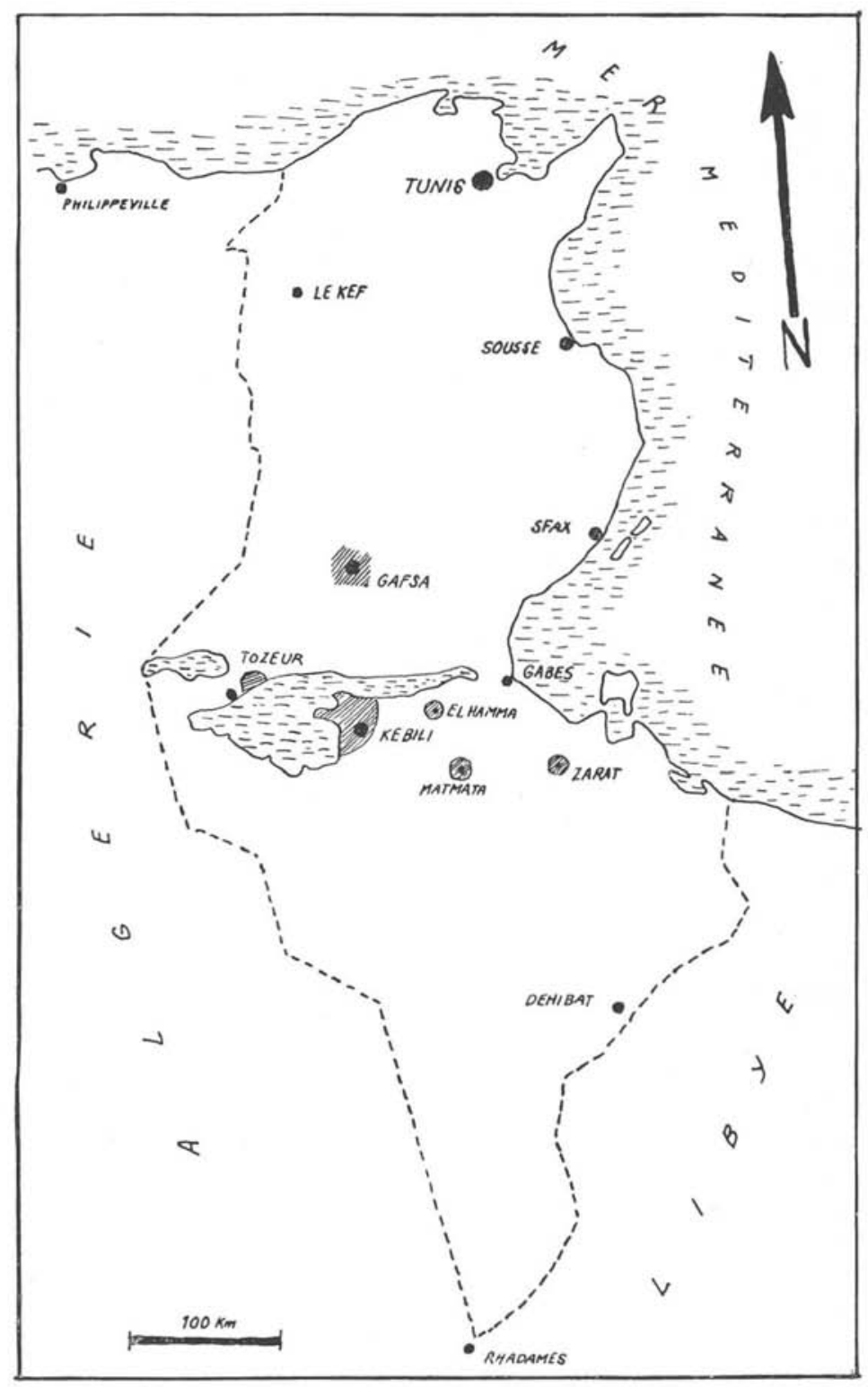




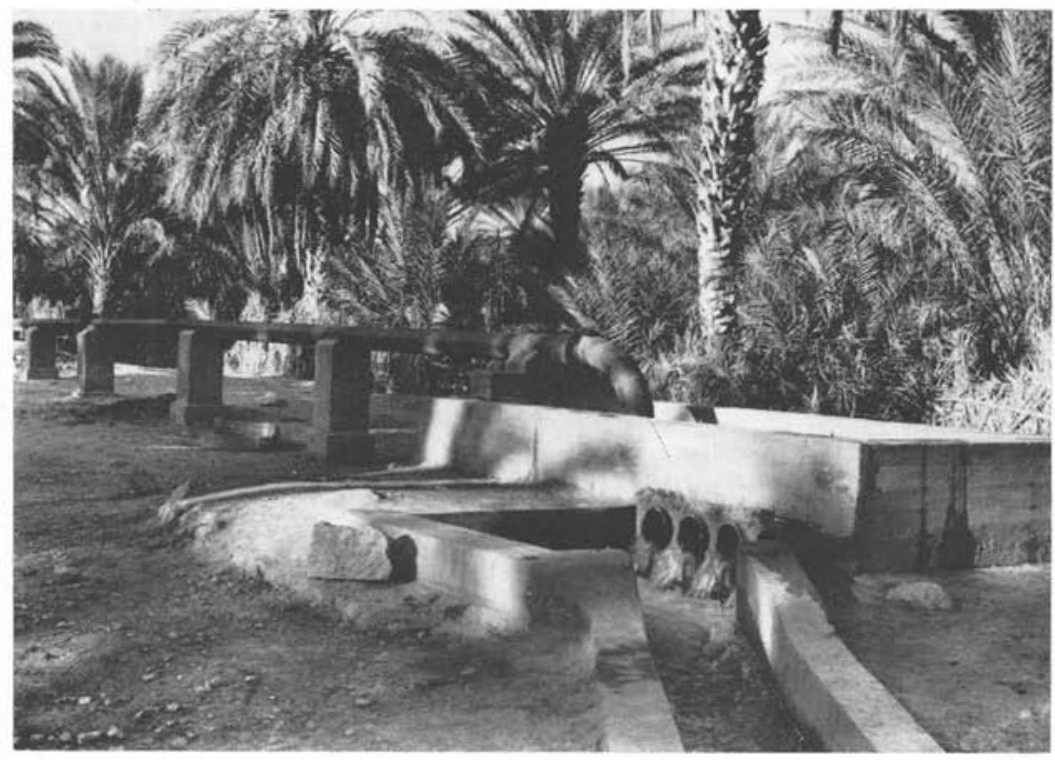

Рното 5. - Antennes de distribution de l'eau d'un réseau d'irrigation. Côte à côte : la « mauvaise » et la «bonne » solutions : conduite ouverte à droite ; conduite fermée à gauche. Le bassin sert au changement de direction et de niveau de l'écoulement. Foyers d'infestation de création récente. Noter la rapidité du courant, qui ne décourage pas la prolifération des bulins

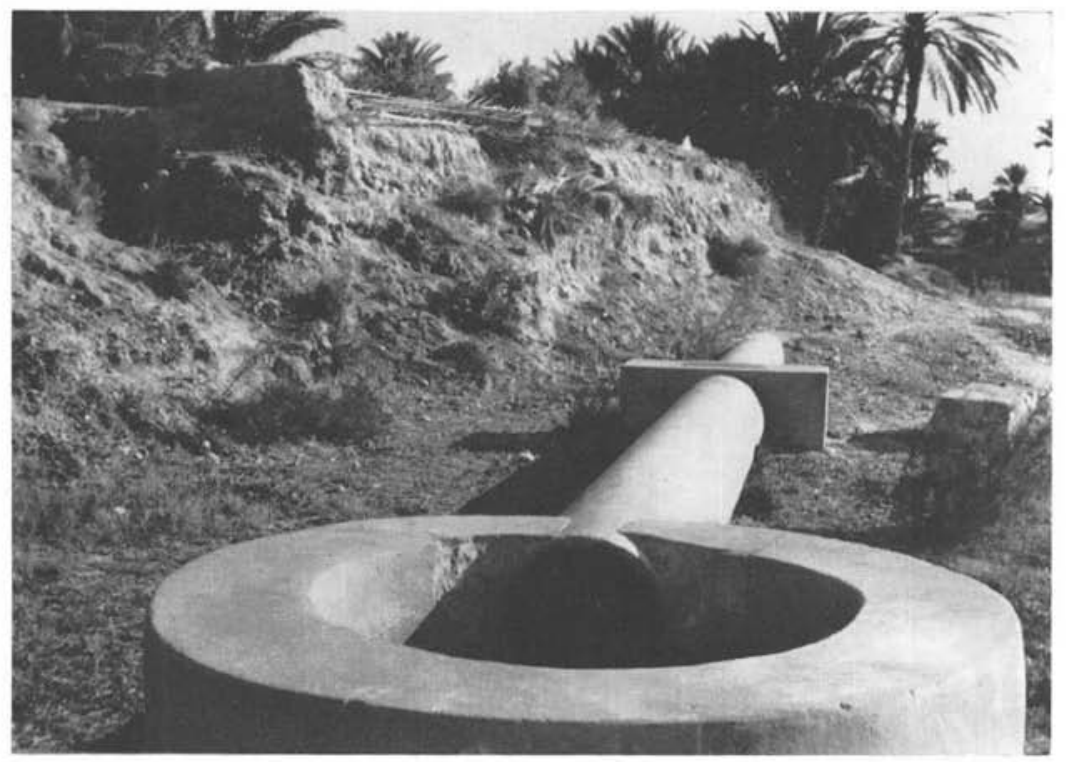

Рното 6. - Une conduite souterraine d'irrigation 
Sur le plan pratique, il convient de faire remarquer que le dépeuplement de certains gîtes est une manifestation sporadique et qu'il ne peut, dans l'état actuel des choses, affecter tant soit peu l'intensité de l'endémie bilharzienne. Il met simplement en évidence le fait que le bulin, qui par ailleurs a des qualités remarquables d'adaptation, est parfois sensible à des variations de l'habitat tellement légères qu'elles échappent à notre observation.

\section{III. - Gîtes récents}

Il s'agit de foyers d'infestation qui, tout comme dans le groupe précédent, sont de création artificielle, consécutive aux pratiques d'irrigation. De création toute récente, ces gîtes sont d'une nature tellement différente de tout ce qui vient d'être vu, que nous avons préféré les grouper dans un chapitre à part.

Nous venons de voir que l'irrigation continue à partir des sources artésiennes ne peut être réalisée par les anciens dispositifs à cause de pertes importantes d'eau par réabsorbation dans le sol tout au long du trajet allant de la source à la zone d'utilisation. Aussi, actuellement, et depuis quelques années seulement, on réalise l'irrigation continue, en amenant l'eau jusqu'au périmètre à irriguer, dans des conduites en béton. La source elle-même est tout d'abord soigneusement déblayée, et le point précis d'où jaillit l'eau coiffé d'un cylindre creux en béton armé appelé buse, d'où part une première conduite de béton souterraine, ou aérienne, qui fait office de séguia principale (photo 4). A partir de cette conduite, partent des antennes secondaires qui s'arborisent à leur tour, tout comme les séguias secondaires du système antique, et qui vont porter l'eau jusqu'à la proximité immédiate des plants à irriguer.

Il s'agit, somme toute, du vieux système d'aqueducs romains, dont on retrouve d'ailleurs les ruines, un peu partout en Tunisie, système qu'on aurait miniaturisé et adapté aux seules fins agricoles (photo 5). Le captage de la source préserve celle-ci d'une manière définitive contre l'ensablement et assure ainsi un débit accru et constant. L'acheminement de l'eau dans des conduites en ciment jusqu'au lieu même de son utilisation élimine les pertes d'eau par réinfiltration. Les multiples avantages d'un tel système font que la pratique de ces dispositifs se généralise dans l'ensemble du Sud tunisien, et tend de plus en plus à remplacer le système ancien d'irrigation intermittente.

Les puits artésiens nouvellement forés en vue de l'irrigation sont systématiquement dotés d'un système de distribution en ciment. Aussi, les incidences sur l'endémie bilharzienne de ces ensembles sont-elles très importantes à élucider.

On admet généralement que la rapidité du courant et le manque de végétation aquatique constituent des facteurs défavorables pour la prolifération des bulins. Or, c'est un fait que l'écoulement d'eau dans les conduites en ciment à pente rigoureusement calculée et à parois lisses est beaucoup plus rapide que dans les séguias en terre dont la pente est imposée par celle du terrain, 
dont le fond s'encrasse continuellement et dont les parois présentent des anfractuosités nombreuses.

Par ailleurs, les conduites en béton ne sont recouvertes, comme seule végétation, que d'une mince pellicule d'algues microscopiques. Tout ceci a fait que l'on a même préconisé la construction de conduites en ciment devant remplacer les séguias en terre comme moyen de lutte contre les mollusques.

Or, nous avons constaté, dans beaucoup d'endroits, sur les parois en ciment de ces constructions, une pullulation de bulins bien plus importante que tout ce que nous avons pu voir dans les gîtes naturels. Les bulins s'accommodent parfaitement d'un courant violent d'une eau pratiquement stérile, puisque nous en avons trouvé en grande abondance à quelques mètres seulement d'un forage artésien portant sur une nappe profonde (photo 5). Les besoins nutritionnels sont parfaitement satisfaits par la mince pellicule d'algues microscopiques qui recouvre les parois des conduites en béton, ce qui remet singulièrement en cause tout ce qui a été dit sur les préférences des bulins pour telle ou telle plante aquatique des gîtes naturels. Les algues de grande taille ne servent en fait aux mollusques que de support mécanique ; il n'y a de préférence qu'en fonction de la microflore qui prolifère sur ces plantes. Cette microflore vient-elle à se développer sur une paroi de béton, les bulins s'accommoderont parfaitement d'un support aussi différent de ceux qui leur sont offerts dans les conditions naturelles. Aussi peut-on se demander si la présence ou l'absence de telles ou telles espèces de micro-algues conditionne ou non la présence ou l'absence de bulins dans un point d'eau donné, si évidemment il n'existe pas par ailleurs d'autre cause inhibitrice concernant ce point d'eau. On conçoit les difficultés que soulèverait l'abord d'un tel problème.

Quoi qu'il en soit, la prolifération des bulins dans ces gîtes inattendus est tout à fait spectaculaire. Les bulins tapissent littéralement les parois de certaines conduites et bassins en béton, sans laisser d'interstices libres, se disposant par endroits sur deux ou plusieurs couches.

On conçoit, dans ces conditions, l'importance de ces nouveaux gîtes en tant que foyers d'infestation bilharzienne. En effet, les conduites d'eau sont jalonnées sur tout leur parcours d'ouvrages en béton, tels que bassins d'accumulation, de dénivellation, de changement de direction, de distribution (photo 5), nécessaires au fonctionnement du système d'irrigation. Tous ces bassins constituent des baignoires idéales pour la population infantile et adulte de la région, et, étant donné la prolifération extraordinairement intense des bulins dans ces bassins, il s'agit de véritables bains infestants. Par ailleurs, les eaux artésiennes, sans cesse renouvelées, donc relativement propres, de ces conduites, sont largement utilisées dans la consommation et à tous les besoins ménagers. Nous voyons donc que, loin d'entraver la transmission de la bilharziose, la construction d'un réseau d'irrigation en béton la favorise, au contraire. 


\section{Perspectives de lutte}

Sans passer en revue de manière détaillée les différentes méthodes de lutte antibilharzienne, voyons quelles sont les possibilités d'action, étant donné les conditions épidémiologiques particulières au Sud tunisien.

Les mesures tendant à interrompre le cycle parasitaire au niveau de l'homme malade nous paraissent peu opérantes. Le traitement en masse des populations atteintes serait certainement sans aucune portée dans les conditions actuelles, étant donné la réinfestation certaine. Le traitement, en tant que mesure prophylactique, ne saurait être envisagé que dans la phase finale d'un programme de lutte, une fois que la destruction des mollusques a été menée à bien. En effet, les médications antibilharziennes sont d'une efficacité inconstante, toxiques, coûteuses.

Dans l'état actuel des choses, on ne devrait entreprendre le traitement que dans quelques rares oasis isolées, où, par suite d'un captage de la source, le foyer infestant a été supprimé, et où le nouveau circuit d'irrigation en béton n’a pas été envahi.

Le contrôle des migrations humaines en tant que mesure tendant à endiguer l'endémie serait à envisager, mais on se doute des difficultés qu'il soulèverait dans la pratique. Le nomadisme constant d'une fraction de la population du Sud tunisien ne représente pas un grand danger, car il s'agit de déplacements en circuit fermé, intéressant toujours les mêmes régions pré-sahariennes.

Autrement plus dangereuse est l'émigration vers le Nord d'ouvriers agricoles qui se produit à la suite d'un appel de main-d'œuvre, dû au développement de programmes d'irrigation dans le Nord tunisien, région à ce jour indemne de bilharziose.

Il serait important de connaître la faune malacologique du Nord tunisien en vue de l'appréciation du danger d'une implantation de la bilharziose.

La construction de latrines, mesure tendant à soustraire les bulins de l'infestation par les Miracidia, n'aura guère d'effet sur l'endémie bilharzienne. Les quelques cycles parasitaires qui ne se feront pas ne pourront guère influencer sensiblement le taux d'endémie si intense de la région. Il est aléatoire de prétendre interdire aux enfants d'uriner pendant qu'ils se baignent, et aux cultivateurs pendant qu'ils travaillent sur leur terrain.

La construction de bains publics serait certainement accueillie avec plus d'enthousiasme par les populations, que celle des latrines. Elle serait plus opérante. Encore faudrait-il prévoir que l'eau utilisée soit dépourvue de cercaires ou qu'elle en soit débarrassée.

Ces mesures ne pourraient donner quelque résultat que si elles étaient menées à bien en même temps que l'éducation sanitaire du public, qui ne doit certes pas être négligée, mais dont il ne faut guère escompter des résultats immédiats. 
L'essentiel de la lutte antibilharzienne réside donc dans la lutte contre les mollusques. Or, celle-ci se présente dans le Sud tunisien dans des conditions particulièrement favorables.

En effet, toute la région atteinte est comprise dans la zone pré-saharienne.

La quantité d'eau à traiter, considérée dans son ensemble, est relativement faible. L'ensemble de la surface submergée servant de support aux bulins est également faible et facilement accessible. Les différents points d'eau naturels ou artificiels, pris isolément, constituent des collections d'eau peu importantes, à faible débit.

Pour entreprendre la lutte contre les bulins, nous sommes donc dans des conditions bien plus favorables que quand il s'agit de vastes terrains marécageux, de rizières comme à Madagascar ou d'un réseau d'irrigation étendu à gros débit comme en Egypte.

La lutte contre les mollusques dans les gîtes naturels et les séguias en terre consistera en curages de la végétation aquatique, qui d'ailleurs sont pratiqués déjà aux fins d'entretien des réseaux d'irrigation, suivies de l'application d'un molluscocide.

En raison de sa non-toxicité aux taux employés pour les plantes et les animaux, et de son prix de revient relativement bon marché, le sulfate de cuivre nous paraît tout indiqué. On tiendra évidemment compte, dans l'établissement du calendrier des opérations, du cycle biologique saisonnier des bulins. C'est immédiatement après l'éclosion des œufs des bulins que la campagne molluscocide est la plus rentable.

Les gîtes à bulins de création récente dans les conduites en béton appellent quelques remarques.

Nous avons vu que le remplacement des canaux en terre par des conduites en ciment ne supprime pas par lui-même la prolifération des mollusques, au contraire. Cependant, les perspectives de lutte dans ces nouvelles conditions s'en trouvent fortement facilitées.

Le captage de sources oasiennes constitue en lui-même la suppression définitive d'autant de gîtes à bulins. Si l'eau est amenée vers les terrains à irriguer par des conduites souterraines, au lieu qu'elle le soit par une séguia ou une conduite aérienne en béton, c'est autant de pris sur l'aire de prolifération des mollusques (photo 6). Chaque fois que cela est possible, les conduites souterraines devraient être préférées aux conduites aériennes. Les ingénieurs hydrauliciens marquent d'ailleurs les mêmes préférences, les conduites ouvertes à l'air étant plus sujettes à détérioration et à encrassement, ce qui implique un entretien. Par ailleurs, les pertes d'eau par évaporation sont évitées avec les conduites fermées.

Par contre, la pose des conduites souterraines est plus coûteuse et suppose une perte de dénivellation entre la source et l'aire à irriguer, ce qui rend parfois le problème insoluble au point de vue hydraulique. Ce dernier inconvénient est évité avec les conduites aériennes, mais fermées, constituées par des tuyaux 
fermés. Avec ce dispositif, on bénéficie d'un prix de pose, identique à celui des conduites ouvertes à l'air libre, on évite l'ensablement et l'encrassement qui se produisent avec ces dernières et, bien entendu, on évite la prolifération des mollusques. Seul, le prix de revient des conduites elles-mêmes sera supérieur à celui des conduites ouvertes en raison des quantités supérieures de béton utilisé. De l'avis des ingénieurs du Génie rural que nous avons consultés, l'utilisation pour l'irrigation de conduites fermées aériennes ou souterraines, à la place de conduites ouvertes, est parfaitement possible et souhaitable, rien que pour des raisons techniques. L'argument sanitaire devrait étayer de tout son poids cette préférence.

La photo 5 montre côte à côte la «bonne » et la « mauvaise » solution : une conduite aérienne fermée (à gauche) et une autre ouverte (à droite), dans un cas où précisément ce dernier dispositif peut être remplacé par le premier.

Nous avons vu qu'en matière de gîtes artificiels en béton, ce sont surtout les différents bassins infestés de mollusques, qui jalonnent les circuits d'irrigation, qui servent de baignoire à la population. En posant dessus une dalle en béton, on en interdirait l'accès, et les bulins ainsi placés dans l'obscurité ne s'y développeraient plus. Cette mesure serait également applaudie par le Génie rural, parce qu'elle éviterait l'encrassement des bassins par le sable, la végétation aquatique et les détritus divers que la population y jette souvent.

Chaque réseau d'irrigation, chaque branche de ce réseau, est un cas d'espèce, avec ses problèmes hydrauliques, topographiques, agricoles, ses possibilités et ses impossibilités de favoriser ou de nuire à la cause sanitaire, de se plier ou non aux exigences sanitaires. Si nous nous sommes attaché à décrire en détail quelques petits problèmes d'hydraulique qui se posent sur le terrain, et quelques-unes des solutions qui pourraient leur être apportées, c'est pour montrer que de telles solutions, acceptables à la fois pour les deux parties, hydraulique et sanitaire, existent dans bien des cas et qu'il convient de les rechercher.

La connaissance des impératifs hydrauliques, tels qu'ils se présentent dans la pratique, sur le terrain, à l'ingénieur hydraulicien, peut seule permettre à l'épidémiologiste de placer le problème sanitaire dans le contexte de la réalité. La collaboration étroite entre Service sanitaire et Génie rural est plus que jamais indispensable, à l'heure où de grands travaux d'irrigation sont entrepris, travaux mettant en place des dispositifs permanents nouveaux, ayant une répercussion très importante sur l'essor de l'endémie bilharzienne. Collaboration à un niveau tel que chacune des parties comprenne suffisamment les problèmes de l'autre, ce qui permettrait de discuter autour d'un problème précis, de suggérer des solutions, et non seulement de proposer des directives générales et d'émettre des vœux aussi judicieux qu'inapplicables.

Ceci dit, il est des cas où une conduite, un bassin, doivent nécessairement demeurer ouverts à l'air libre, soit de par sa fonction même : abreuvoirs d'animaux, lavoirs; soit par suite d'impératifs techniques; soit parce qu'un système, étant déjà construit de telle manière, on trouve trop coûteux de le recons-

Ann. de Parasitologie, T. XXXVII, N ${ }^{\circ} 3 .-1962$. 
truire en tenant compte des critères sanitaires. Dans ces cas, et dans tous les cas où les données d'un problème hydraulique sont telles qu'aucune modification de caractère permanent ne peut être apportée en vue d'écarter le danger bilharzien, il ne reste rien d'autre à faire que d'appliquer à ces gîtes les méthodes de lutte habituelles: curage et molluscocides chimiques. Il convient de souligner que nous sommes là encore dans des conditions de lutte particulièrement favorables. Il suffit, en effet, de se servir des dispositifs utilisés pour le fonctionnement de ces réseaux d'irrigation, pour régler le débit ou assécher complètement telle ou telle partie du circuit. Pour venir à bout de cette « bilharziose d'aquarium », et pour la surveiller de près, nous sommes placé dans les conditions d'une expérimentation de laboratoire. C'est plus que n'a jamais pu espérer un épidémiologiste en matière de bilharziose.

Pour toutes les raisons qui viennent d'être évoquées, nous pensons que, tant les gîtes naturels, anciens, que les gîtes artificiels de création récente, offrent des conditions exceptionnellement favorables à la lutte contre les mollusques. Par la transformation définitive des points d'eau à l'occasion de l'application des méthodes modernes d'irrigation, conjuguée avec l'utilisation de molluscocides, nous pensons qu'il est possible d'obtenir en Tunisie ce résultat très rare en matière de lutte antibilharzienne : l'éradication des bulins de toute la région infestée et le maintien de ce résultat moyennant une surveillance périodique.

Nous pensons que l'effort mérite d'être tenté, à la condition qu'il s'agisse d'un programme de lutte rationnel et systématique, appliqué à toute la région intéressée, toute action parcellaire et incomplète ne pouvant donner aucun résultat à longue échéance. Nous pensons que la lutte devrait être entreprise le plus rapidement possible: en effet, le système d'irrigation qui a fonctionné sans changement aucun depuis des centaines d'années, assurant la pérennité, mais aussi la fixité des foyers bilharziens, est en train de se transformer rapidement. C'est le moment où une coopération étroite entre le Génie rural et les Services sanitaires peut aboutir à l'élimination de nombreux foyers. Sans quoi, l'expansion de l'irrigation s'accompagnera forcément de la création de foyers infestants nouveaux de plus en plus nombreux, du type particulièrement dangereux, que nous venons d'étudier, et par conséquent d'une aggravation de l'endémie bilharzienne dans les régions déjà atteintes. La culture irriguée se développant également dans les régions jusqu'à présent indemnes, il est probable qu'on assistera à l'extension de la bilharziose à ces régions, ainsi que cela s'est déjà souvent produit dans d'autres pays dans des conditions semblables.

Toutes les conditions requises pour une expansion de la bilharziose sont réalisées aujourd'hui en Tunisie. Cependant, l'occasion nous est en même temps offerte d'exploiter ces nouvelles conditions dans une lutte contre les mollusques vecteurs, lutte qui ne manquerait pas de mener à une victoire définitive sur une endémie plusieurs fois centenaire. 


\section{Conclusions}

L'endémie bilharzienne en Tunisie est représentée par quelques foyers bien isolés, disséminés dans la région pré-saharienne du Sud du pays, et présentant un taux d'infestation de l'ordre de $50 \%$ de la population.

Les habitats des mollusques responsables appartiennent à trois catégories :

1) les gîtes ancestraux naturels, oueds et sources d'oasis, réservoirs de virus de tout temps;

2) les gîtes créés par l'homme à l'occasion de la pratique séculaire de la culture irriguée : canaux d'irrigation en terre, et

3) les gîtes de création toute récente, créés par les méthodes modernes d'irrigation, constitués par des conduites et des bassins en béton. Chacun de ces types de foyers importants possède ses caractéristiques épidémiologiques particulières et évolue, à la suite de modifications des données hydrauliques, d'une manière qui lui est propre. Les méthodes de lutte, pour être rationnelles et de longue portée, doivent tenir compte de ces particularités.

L'endémie bilharzienne est un exemple de la grande stabilité d'un équilibre biologique dans la nature. La bilharziose, qui sévit aujourd'hui inchangée en Egypte, y était déjà du temps des pharaons, puisqu'on a découvert des parasites dans le corps des momies égyptiennes. La bilharziose tunisienne porte en elle certainement la même capacité de stabilité. Cependant, cette stabilité n'est qu'apparence, car elle résulte à tout moment de la stabilité d'un équilibre assurant l'accomplissement du cycle du parasite, équilibre qui dépend de bien de facteurs, généralement stables, il est vrai, pouvant agir sur l'un des maillons homme ou mollusque de la chaîne de transmission. L'un de ces facteurs se modifie-t-il, comme c'est le cas dans les transformations de l'habitat des mollusques qui viennent d'être décrites, l'équilibre biologique dans son ensemble s'en trouve affecté et se déplace vers une expansion ou une régression de l'endémie.

De grands travaux d'irrigation ont été entrepris ces dernières années en Tunisie, selon des méthodes modernes, jusqu'ici jamais appliquées à ce pays. Il s'ensuit une modification profonde des données qui conditionnent l'endémie bilharzienne. L'équilibre se trouve rompu en faveur de cette dernière, tant en ce qui concerne son intensité que son expansion géographique.

Cependant, ces modifications sont telles qu'elles offrent à la lutte contre les mollusques des conditions exceptionnellement favorables, jusqu'à permettre d'espérer leur éradication définitive. L'heure actuelle nous paraît donc être un moment unique pour entreprendre cette lutte. 


\section{Bibliographie}

1. Coumbaras (A.). - Arch. Inst. Pasteur Tunis, 1960, 37, 313 et 1961, 38, 255.

2. Gaud (J.). - Bull. Org. Mond. Santé, 1958, 18, 751.

3. Nicolle (Ch.) et Gobert (E.). - Arch. Inst. Pasteur Afrique du Nord, 1921, $1,231$.

4. Gobert (E.). - Arch. Inst. Pasteur Tunis, 1934, 23, 348.

5. Versiell (C.). - Arch. Inst. Pasteur Tunis, 1957, 34, 167. 